\title{
Type I (Hannis - Steinthal) Capitellar Fracture: Case Report
}

\author{
V.M. Mutiso, MBChB, MMed (Surg), FCS, ECSA, Lecturer, Department of Orthopaedic Surgery, \\ University of Nairobi, Medical School, Kenyatta National Hospital, P.O. Box 19681-00202, Nairobi, Kenya, \\ Email:mutisovm@yahoo.com
}

\begin{abstract}
Capitellar fractures of the elbow present with pain and swelling of the elbow following a fall on an outstretched arm and are rare injuries comprising $0.5-1 \%$ of all elbow fractures (1). This is a case report of an adult female who sustained this injury following a fall on her outstretched arm. CT scans revealed a Type I capitellar fracture of the right elbow. Open reduction and internal fixation was done with a functionally good result. Because of the cartilaginous nature of the capitellum humeri these injuries are easily missed on radiography. One must have a high index of suspicion especially when elbow movement is noticeably restricted. These injuries are discussed and the literature reviewed. Although controversial, treatment is mainly operative. One should carefully review radiographs of patients who present with injuries of the elbow with pain, swelling and markedly restricted joint range of motion and whose mechanism of injury(fall on an outstretched arm) is consistent with capitellar fracture. CT scans often clinch the diagnosis. Type I fractures may be treated conservatively but Type II injuries require operative intervention.
\end{abstract}

\section{INTRODUCTION}

The elbow joint is often injured following falls as the individual usually puts the arms out to break the fall. Any part of the elbow joint can be injured in these circumstances however capitellar injury is relatively rare. Because a large portion of the capitellum is cartilaginous the injury is also more difficult to spot on plain radiographs. A case of capitellar fracture is presented and the literature reviewed.

Anatomy: The elbow joint is a synovial joint of the hinge variety. The distal humerus articulating surfaces include the trochlear - which articulates with the ulna, and the capitellum, which articulates with head of the radius $(2,3)$. The capitellum forms a portion of a sphere. It projects forwards and downwards where its lower border lies at the distal extremity of the humerus. The inferior articular surface of the capitellum does not extend posteriorly. The radial head articulates with the anterior surface when in flexion and with the inferior surface when in extension (1-3). The radial fossa, a depression on the anterior humerus just above the capitellum accommodates the margin of the radial head when the elbow is acutely flexed $(3,4)$.
Mechanism of injury: Fractures of the distal humerus articular surfaces usually includes fracture of the capitellum, trochlear, or both. There is little soft tissue attachment to the capitellum and thus no avulsion forces. The forces are mainly compressive and shearing (1). The capitellum is fractured usually following a fall on the outstretched upper limb with the elbow in slight flexion or directly onto the elbow with the elbow in full flexion. Force is transmitted through the radial head and thus an association with fracture of the radial head. Other associated injuries include the medial (ulna) collateral ligament, dislocation of the elbow, tear of the interosseuos membrane and distal radioulna disruption (2-5). The fragment is usually displaced anteriorly but may be displaced posteriorly.

Classification: There are numerous classifications of capitellar fractures. These include;

1. Classification by Mehne \& Jupiter and by Kocher and Lorenz (1).

2. Conventional classification divides these injuries into two types: $(2,5,6)$.

- Type I (Hahn - Steinthal):The fracture involves a large part of the osseous portion of the capitellum and may contain part of; the adjacent lip of the trochlear. 
- Type II (Kocher -Lorenz): Involves articular cartilage with very little bone attached.

- Type III:Comminuted

(Wilson described a third type in which the articular surface is driven proximally and impacted into the osseous portion).

3. Brian and Morley classification is almost identical and is as follows (7):

Type I: Complete osteochondral fracture

Type II: Superficial osteochondral fracture

Type III: Comminuted

Type IV: Coronal shear fracture (described by McKee involving the capitellum and a portion of the trochlear)

Treatment: Both operative and non operative modes of management have their advocates depending on severity.

Non displaced fractures can be treated conservatively with splintage whilst for fractures with displaced fragments there are the following options:

- closed reduction

- open reduction and internal fixation

- open reduction with excision of fragments.

The radial fossa must be cleared of all fragments for the elbow to regain full range of flexion.

\section{CASE REPORT}

XX a 28 year old unmarried International Aid worker stumbled and fell on her partially flexed right arm during a trip to Addis Ababa in Ethiopia. She felt a sharp pain in her right elbow with swelling and total limitation of movement. She was attended to by a local orthopaedic surgeon who splinted the elbow in flexion.

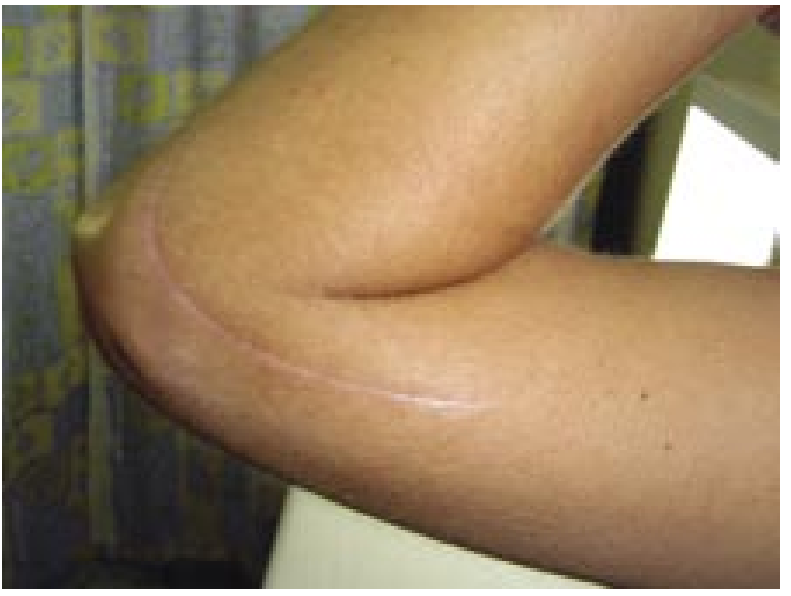

Post-operative flexion.
On her insistence, she was evacuated to Nairobi ,Kenya. On review, this was an anxious young adult female.

Her right elbow was held in flexion by a splint and on removal of the splint the elbow was found to be acutely tender, swollen, bruised and discoloured. She would not allow either pressure or active movement of the joint. Initial plain radiographs were difficult to interpret. Subsequent ones and a CT scan of the elbow revealed an anteriorly displaced Type I (Hannis Steinthal) capitellar fracture of the elbow joint.Following discussion of management options and consultation with her orthopaedic relative, open reduction and internal fixation was agreed upon.

This was done with AO small fragment $3.5 \mathrm{~mm}$ screws and Kirshner wire through a lateral approach, taking care not to injure the posterior interosseous nerve. Post operatively the elbow was held in $90^{\circ}$ of flexion with slight pronation. Vacuum drain was removed after 24 hours and she was discharged on the $3^{\text {rd }}$ post operative day.

She was reviewed two weeks postoperatively at which time the splint was removed. Check X-rays showed no alteration from the immediate postoperative radiographs. Gentle elbow therapy was commenced.

At six weeks post operatively, a further check X-ray revealed the operative position to have been maintained and elbow therapy was stepped up. As the pain and swelling subsided, she had an elbow joint range of motion sufficient to allow reasonable activities of daily living. Soon after this review she relocated back to her home in the United States and was lost to follow up.

By the time of her departure, she had $30^{\circ}-130^{\circ}$ of flexion and extention with $70^{\circ}$ of pronation - supination. There was no evidence of a avascular necrosis of the fractured fragment and the elbow was painfree.

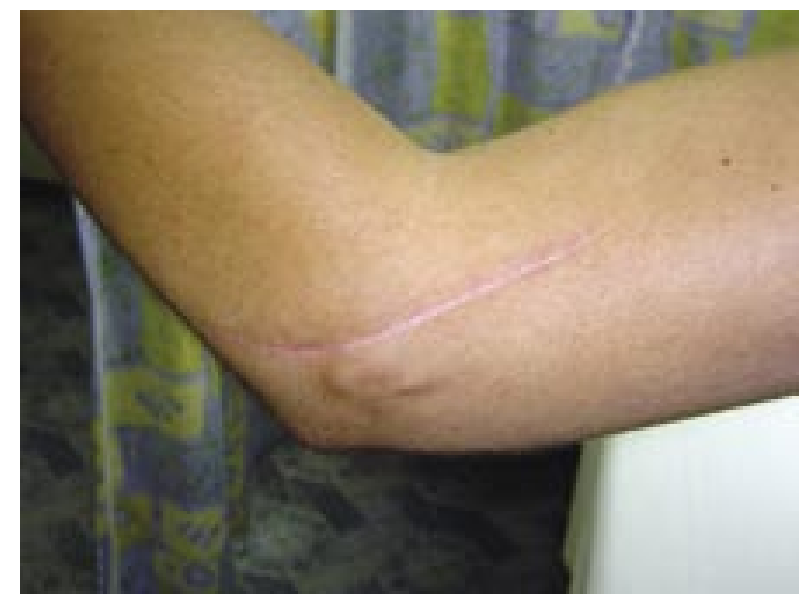

Post-operative extension. 

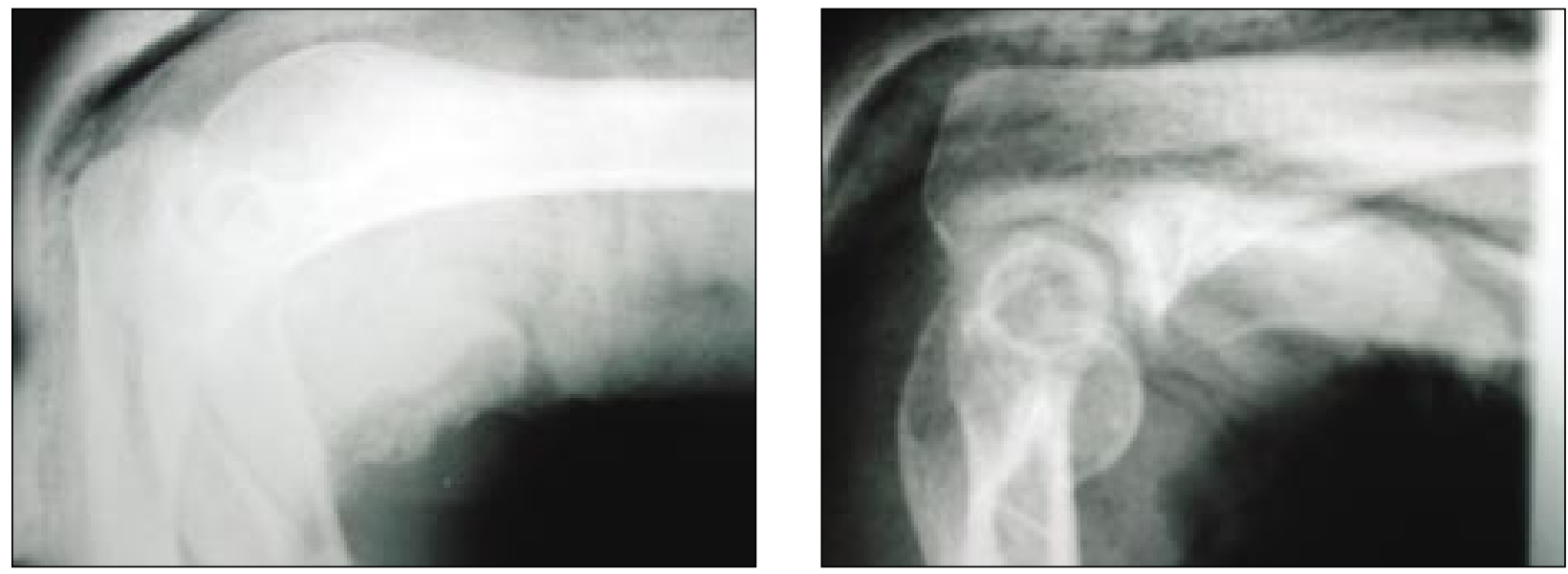

Plain X-ray lateral view
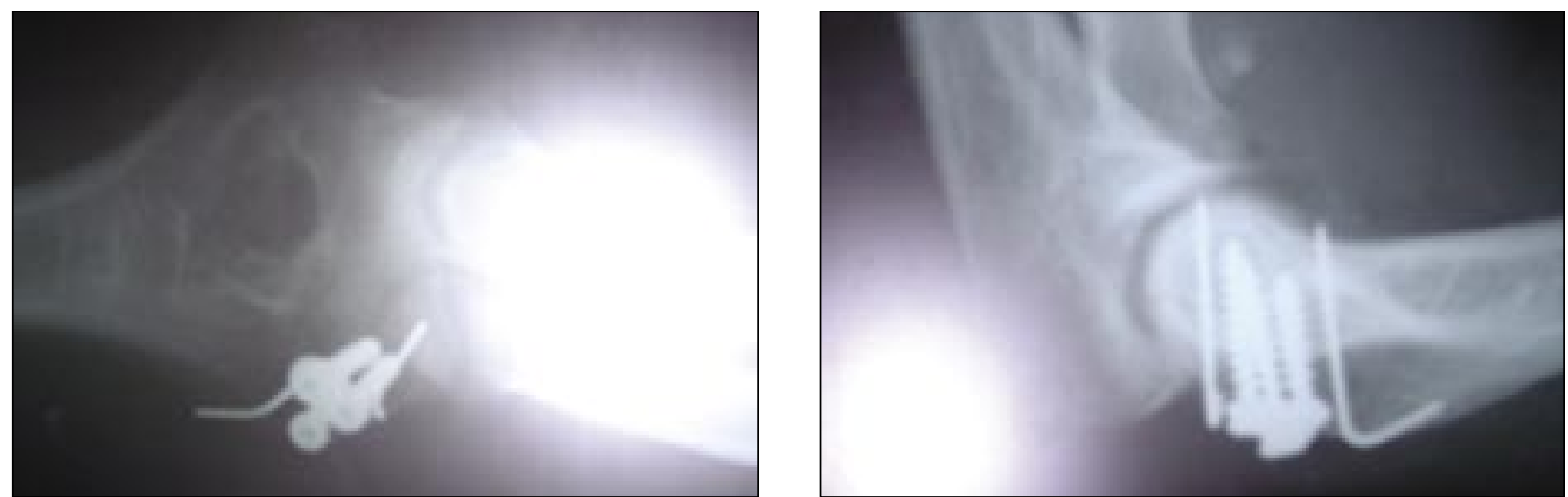

Check X-rays after twelve weeks
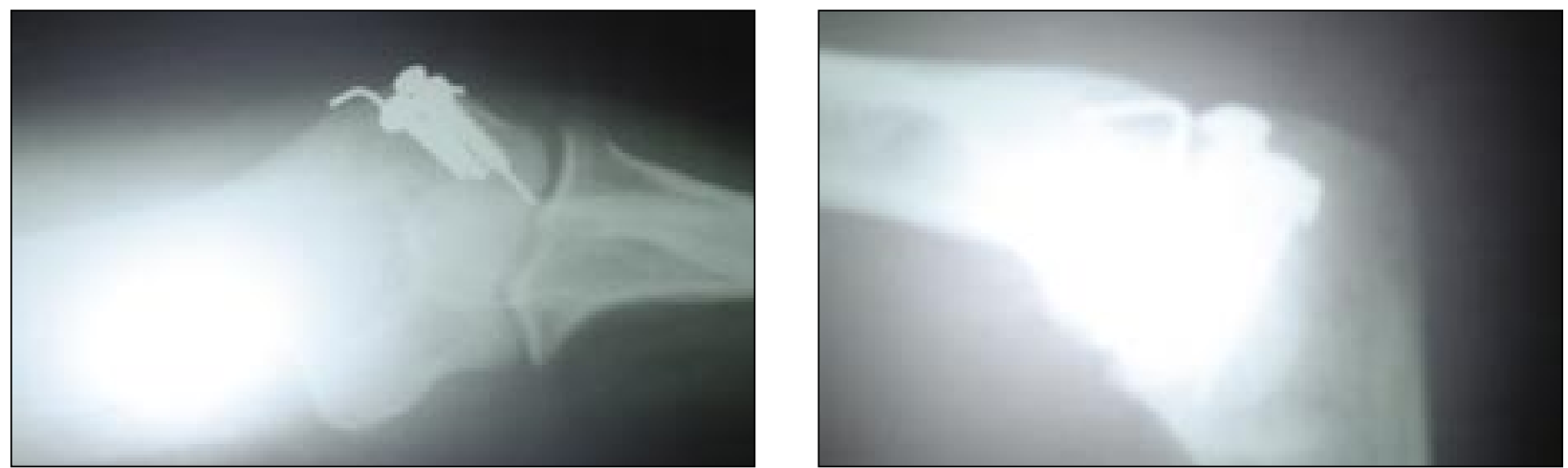

Post-operative $X$-rays

\section{DISCUSSION}

Capitellar fractures are rare with an incidence of $0.5-1 \%$ (1). Some authors allege a greater incidence in women. They were first described y Kane \& Hahn in 1853.

There is usually a history of fall with pain and swelling of the elbow due to distention of the joint by haematoma. The fall is usually on an outstretched arm or a flexed elbow. Impingement results in reduced joint range of motion which has been described by Alvanez
\& Colleague as Type I: Block to flexion and Type II: Block to extention (6).

There is limitation of flexion and extension but pronation and supination are reasonably well preserved. Rarely the fragment is displaced posteriorly $(1,3)$.

Radiographs do not always clearly show the injury especially on anterior posterior views. Lateral views tend to be more informative but one needs a high index of suspicion to detect the injury. One cannot see the true size of the fragments because of the the cartilage. This 
is especially so when the capitellar fragment is almost entirely cartilaginous. CT scans clarify the situation well and are recommended. CT scans should be done for accurate diagnosis of size, origin and displacement of the capitellar fracture.

Some surgeons advocate conservative measure with splinting of the elbow. Obviously displaced fragment will affect joint range of motion. Undisplaced fraactures should be splinted at $90^{\circ}$ for two weeks followed by gentle physiotherapy. Avoiding extreme closed manipulation is advocated by some surgeons. Reduction and maintenance of the reduced fragment may be difficult.

One should look for associated injuries of the radial head reported to have a $20 \%$ incidence in these injuries and ligamentous structures around the elbow $(1,3,5,6)$. Excision of fragments is advocated by some especially if the fragment is entirely cartilaginous.

A significant group of surgeons advocates open reduction and internal fixation (1,3,5,8-10).The aim being anatomical restoration. Operative approach varies but the lateral is popular.The operative approaches vary and may also be anterior, lateral or postero - lateral.Care needs to be taken to avoid injury to the posterior interrosseous nerve where lateral approaches are used.

Fixation can be done with wires and screws (A.O. Screws Smillie or Herbert). Avascular necrosis does not seem to be a long-term complication. Elbow physiotherapy is important. It is commenced early following excision of fragments or stable fixation. Union of capitellar fractures has been reported after both closed and open reduction even though the capitellum fragments had little soft tissue attachment and vascularity.

Non-operative management is advocated for nondisplaced fractures. Generally closed reduction is done with the elbow in extention and immobilized in flexion for 4-6 weeks. Pronation results in radial head fixation of the capitellar fragment (11).

Operatively the choice of excision is reasonable when the fragments are small and multiple and/or they have little subchondral bone (Type II).

When both the capitellum and radial head are damaged, one can reconstruct the capitellum and excise the radial head $(1,3,5)$.

In cases of late diagnosis it may be best to excise the radial head to regain joint range of movement.

Avascular necrosis on X-ray does not seem to markedly impair function of the elbow. Possibly revasculaisation of the subchondral bone occurs with creeping substitution. The load across the radiocapitellar joint is insufficient to cause collapse and discontinuity.

For activities of daily living (ADL'S) one requires 30 $130^{\circ}$ of flexion and extention (normal $0-150^{\circ}$ ) and $50^{\circ}$ of pronation and supination (normal pronation is $80^{\circ}$ and supination $\left.85^{\circ}\right)(5)$. The articular anatomy plays an important part in contributing to elbow stability while the medial collateral ligament is the prime stabilizer of the joint.

\section{CONCLUSION}

It is likely that a lot of capitellar fractures are missed owing to them having a large cartilaginous component. Orthopaedic surgeons should have a high index of suspicion. When recognized early and appropriately treated, outcomes are satisfactorily and allow activities of daily living.

\section{REFERENCES}

1. Rockwood \& Greens. Fractures in Adults Vol. 1, $4^{\text {th }}$ Edn. Vol. $1 \mathrm{Ch}$. 14. Fracture \& Dislocations of the elbow by Robert Hotchkiss. Lippincott-Rower Publ. Philadelphia. 1996.

2. R.J. Last. Aids To (Pocket Anatomy ) $12^{\text {th }}$ Edition Bailliere,Tindall \& Cassell 1962.

3. Orthopaedic Knowledge Update 7 Home Study Syllabus. American Academy of Orthopaedic Surgeons, Edited by Kenneth K Koval MD Jaypee $1^{\text {st }}$ Indian Edition 2004.

4. W.J. Hamilton. Textbook of human anatomy, $2^{\text {nd }}$ Ed. Macmillan Brass. 1976.

5. Janos P Ertl MD, University of California. Capitellar Fracture. eMedicine, July $9^{\text {th }} 2004$

6. AlvarezF., Patel M.R. and Nimberg G. Fracture of the capitulum humeri. J. Bone \& Joint Surg. 1975; 57 A: 1093-1096.

7. Bryan R.S. and Morrey B.F. Fracture of the distal humerus in Morrey B.F. The elbow and its disorders. Philadelphia Saunders.1985; 302-339.

8. Fowles J.V. and Kassab. M.T. Fracture of the capitellum humeri. J. Bone \& Joint Surg. 1974; 56A: 794-798.

9. Conn J. and Wade P.A. Injuries of the elbow: A ten year review. J. Trauma. 1961; 1: 248-268.

10. Collert S. Surgical management of fracture of the capitellum Humeri. Acta. Orthopaed. Scan. 1977; 48: 603-606.

11. Dushuttle R.P., Coyle M.P., Zawadsky J.P. and Bloom H. Fractures of the capitellum. J. Trauma. 1985; 25: 317-321.

12. Simpson L.A. and Richards R.R. Internal fixation of a capitellar fracture using Herbert screws. Clin. Orthop. 1986; 209: $166-168$ 